\title{
LA EPIGRAFÍA ROMANA DE AUGUSTA EMERITA MÁS ALLÁ DEL MUSEO: DIGITALIZACIÓN, MODELIZACIÓN 3D Y DIFUSIÓN A TRAVÉS DE DISPOSITIVOS MÓVILES
}

\section{ROMAN EPIGRAPHY OF EMERITA AUGUSTA BEYOND THE MUSEUM: DIGITIZATION, 3D MODELING AND DISSEMINATION THROUGH MOBILE DEVICES}

Manuel Ramírez-Sánchez

Universidad de Las Palmas de Gran Canaria. Instituto Universitario de Análisis y Aplicaciones Textuales

manuel.ramirez@ulpgc.es

José Pablo Suárez Rivero

Universidad de Las Palmas de Gran Canaria. Instituto Universitario de Microelectrónica Aplicada

josepablo.suarez@ulpgc.es

Haridian Guerra Soto

Universidad de Las Palmas de Gran Canaria

haridianguerrasoto@gmail.com

\section{Resumen}

Este trabajo ofrece la experiencia de innovación científica de digitalizar y modelar en 3D un conjunto de cincuenta y nueve inscripciones romanas del Museo Nacional de Arte Romano (Mérida, Badajoz), a través de una tecnología de bajo coste, basada en el modelado digital de 
imágenes en movimiento (Structure from Motion, SfM). Su difusión a través de internet y el desarrollo de una aplicación propietaria para su visualización en smartphones y tablets con sistema operativo Android. Los resultados obtenidos permiten comprobar el potencial de estas tecnologías para incrementar la visibilidad y difusión del patrimonio arqueológico que se conserva en los museos.

Palabras clave: Epigrafía. Inscripciones. Digitalización. 3D. Patrimonio digital. Museos. Aplicaciones móviles.

\begin{abstract}
This paper offers the experience of scientific innovation of digitizing and 3D modeling a collection of fifty-nine Roman inscriptions from the National Museum of Roman Art (Mérida, Badajoz) through a low-cost technology based on Structure from Motion (SfM). The project includes the dissemination through the Internet and the development of a proprietary application for viewing on smartphones and tablets with Android mobile operating system. The results confirm the potential of these technologies to increase the visibility and dissemination of archaeological heritage preserved in museums.
\end{abstract}

Keywords: Epigraphy. Inscriptions. Digitization. 3D. Digital Heritage. Museums. Mobile Devices.

\title{
1. INTRODUCCIÓN ${ }^{1}$
}

El desarrollo que han experimentado en la última década las tecnologías relacionadas con el procesamiento digital de las imágenes y su difusión en internet ha sido espectacular, sobre todo a raíz del impulso de la llamada web social, que ha hecho pivotar el valor de estas herramientas, convirtiéndolas en poderoso instrumentos para la socialización del conocimiento. En este contexto, expresiones que hasta ahora eran poco utilizadas, como la de epigrafía digital, han pasado a convertirse en una expresión refugio que sirve para situar a

\footnotetext{
${ }^{1}$ Este trabajo ha sido realizado en el marco del proyecto Inscripciones romanas de Augusta Emerita en 3D: del Museo a los dispositivos móviles (Ref. FCT-14-8668), financiado por la Fundación Española de Ciencia y Tecnología del Ministerio de Economía y Competitividad. Además, tanto la redacción de este trabajo como las revisiones posteriores del mismo se han realizado durante una estancia de investigación realizada por el primero de los firmantes en el Laboratorio di Cultura Digitale de la Università di Pisa, financiada por el "Programa Salvador de Madariaga 2015" de estancias de movilidad de profesores e investigadores españoles en centros extranjeros del Ministerio de Educación, Cultura y Deporte (Ref. PRX15/00462).
} 
quienes, desde hace ya tiempo, nos dedicamos a la docencia e investigación de esta materia en la universidad (Ramírez-Sánchez, 2016: 365-392).

Quienes tenemos ya una cierta edad miramos hacia atrás y podemos comprobar cómo en solo dos décadas nuestro oficio ha sufrido una profunda transformación. Nadie podría imaginar, por ejemplo, el desarrollo que iban a alcanzar aquellos primeros recursos digitales para la investigación y divulgación del patrimonio epigráfico que comenzaron a aparecer en la década de los noventa del pasado siglo. Pensemos, por ejemplo, en la base de datos epigráfica desarrollada por Gèza Alföldy y su equipo de colaboradores en la Universidad de Heidelberg, primero accesible solo desde los propios ordenadores del Seminario que él dirigía y que, a fines del siglo XX, daba el gran salto de estar a disposición de cualquier investigador del mundo a través de internet ${ }^{2}$.

Con posterioridad a aquella surgieron otras bases de datos epigráficas, algunas especializadas en un país o región del antiguo Imperio romano, otras especializadas en determinados tipos de inscripciones (Ramírez-Sánchez, 2003: 275-301). Podemos mirar atrás, con la perspectiva que nos dan los años transcurridos y confirmar que, con esta nueva concepción de los corpus epigráficos, ahora en formato online, incurrimos en el mismo error en el que cayeron los pioneros de la epigrafía científica en la segunda mitad del siglo XIX.

En efecto, en estas primeras bases de datos epigráficas hemos concedido más importancia al texto que al propio soporte. Estas bases de datos epigráficas cuyo uso está tan extendido entre los especialistas de tal suerte que el primer paso de cualquier labor investigadora se inicia con una búsqueda simple o avanzada en ellas, han privilegiado la lectura y transcripción de las inscripciones frente a los datos físicos de los objetos o monumentos en los que estas inscripciones están realizadas (Ramírez-Sánchez, 2010: 1541). Podemos decir que, en el desarrollo inicial de la epigrafía digital, el texto también ha relegado a un plano secundario al propio monumento, algo que se puede comprobar ante la ausencia de fotografías en la mayoría de los registros de estas bases de datos. Es más, cuando estas fotografías hacen acto de presencia en algunos registros, su calidad es tan deficiente que prácticamente tienen un carácter testimonial y carecen de cualquier valor para el investigador avanzado, que debe seguir recurriendo a los tradicionales corpus epigráficos publicados en papel para encontrar buenas reproducciones fotográficas.

Paradójicamente, es más frecuente encontrar buenas reproducciones fotográficas en plataformas de la llamada Web 2.0 o Web Social, como Flickr, en la que entusiastas amantes de la Arqueología Clásica, e incluso algunos docentes e investigadores, comparten sus fotografías en sus álbumes personales, y en ocasiones también, a través de las decenas de grupos y listas de inscripciones generados en esta conocida red social para compartir

\footnotetext{
${ }^{2}$ Epigraphische Datenbank Heidelberg. Accesible desde http://edh-www.adw.uni-heidelberg.de/home.
} 
fotografías (Elliott, 2014: 83). Sin ánimo de realizar aquí un recorrido exhaustivo por las galerías y grupos de Flickr que ofrecen excelentes fotografías de inscripciones, baste señalar aquí el grupo Visibile Parlare-Ancient Inscriptions, creado en mayo de 2008 por I. Traube, que en el momento de redactar estas líneas cuenta con 6.259 fotografías, distribuidas en catorce temas, y 531 miembros; o el grupo Visibile Parlare-Medieval Inscriptions, creado por el mismo usuario en enero de 2010, que cuenta con 1.164 fotografías y 91 miembros $^{3}$. El mayor inconveniente de muchas de estas fotografías es que sus usuarios las han publicado en sus perfiles con el mayor grado de protección (todos los derechos reservados), por lo que muchos de estos recursos no pueden ser aprovechados por las propias bases de datos epigráficas. Por el contrario, los usuarios de estas redes sociales, a través incluso de los comentarios de las fotografías, enlazan con estas bases de datos y copian las transcripciones junto a las fotografías, proporcionando así un excelente espacio de trabajo colaborativo y de socialización del conocimiento. Sería recomendable que muchas de estas fotografías tuviesen alguna de las licencias Creative Commons que permiten su difusión en otros proyectos. De hecho, muchas de las fotografías publicadas en Flickr que cumplen estos requisitos son utilizadas en proyectos como Wikipedia y Wikimedia Commons, por lo que muchos usuarios de Flickr son los principales suministradores de recursos fotográficos de estos proyectos colaborativos (Fuster Morell, 2011: 229-248).

Esta filosofía ha llegado también al ámbito de las bases de datos epigráficas, a través del consorcio EAGLE y su transferencia a Europeana (Orlandi et al., 2014), permitiendo así, que el caudal de información disponible en las bases de datos epigráficas pertenecientes a este consorcio, puedan ser rentabilizadas por Wikimedia Commons a través de una pasarela denominada EAGLE Mediawiki (Liuzzo et al., 2014: 187-201). A través de proyectos en los que puedan interactuar agentes diversos como grupos de investigación epigráfica, ingenieros informáticos, epigrafistas que se beneficien de los recursos, o usuarios finales y el público en general, será posible el desarrollo de proyectos aún más ambiciosos.

Pero sin lugar a dudas, uno de los campos en el que más ha avanzado la tecnología digital en los últimos años ha sido en el de la fotografía digital. Observando varios trabajos recientes en los que se explica qué es la epigrafía digital (Bodel, 2012: 287-291; Elliott, 2014: 83), se puede comprobar cómo la obsolescencia de la información en esta parcela hace que estemos más cerca de campos como la Ingeniería Gráfica o las Ciencias de la Documentación que de los campos habituales en los que nos hemos movido hasta la fecha los estudiosos de las inscripciones. Más aún, si retrotraemos nuestra mirada hasta la primera aplicación de la tecnología digital y la modelización 3D, hace alrededor de once años, momento en el que un grupo de epigrafistas e ingenieros italianos publicó los resultados de una experimentación con

\footnotetext{
${ }^{3}$ El grupo Visibile parlare-Ancient Inscriptions recoge inscripciones en alfabeto latino de época antigua (hasta fines del siglo V d. C.): https://www.flickr.com/gropups/medievalinscriptions.
} 
láser escáner para poder leer inscripciones depositadas en el Museo Lapidario Maffeiano de Verona (Buonopane et al., 2005: 373-388).

A raíz del rápido desarrollo que ha experimentado el tratamiento digital de las fotografías, así como el perfeccionamiento de software, que permiten modelar en 3D las inscripciones sin necesidad de utilizar costosos escáneres láser; en los últimos años estamos asistiendo a una auténtica revolución que, en cierta medida, pronostica una democratización de estas técnicas. Además, la tecnología 3D conforma un paso de gigante frente a la fotografía tradicional de carácter bidimensional, introduciendo la investigación del patrimonio epigráfico en el ámbito de objetos digitales tridimensionales. Se le otorga al epigrafista la cualidad de interactuar con inscripciones digitales, mejorando la percepción de la naturaleza de los objetos escritos y, en muchos casos, incluso, ayudando a obtener una lectura más precisa de las inscripciones, con un grado de realismo únicamente superable por su estudio directo.

Los primeros trabajos derivados de esta novedosa línea de investigación han sido publicados en los últimos años, mediante técnicas de modelización 3D de calcos epigráficos (Barmpoutis et al., 2010: 989-998) o por trabajos experimentales realizados sobre monumentos epigráficos de época tardoantigua (Lerma y Muir, 2014: 311-318). Más aún importante para los epigrafistas son las técnicas innovadoras del Morphological Residual Model (MRM), desarrollado por el portugués Hugo Pires, aplicable para estudiar restos arqueológicos en superficie, en grabados rupestres (Pires et al., 2014: 133-144) o en inscripciones, pudiendo restituirse textos originalmente escritos sobre la piedra que, por la erosión, se han perdido para un examen autóptico (Correia Santos et al., 2014a: 197-224; 2014b: 437-454). ${ }^{4}$

En 2014 se inicia el proyecto Epigraphia 3D, en el que se inscribe este artículo, a través de la experimentación con la técnica denominada Structure from Motion (SfM), medio de bajo coste, para la documentación y difusión del patrimonio epigráfico, en un campo de indudable interés para avanzar con las colecciones digitales en 3D de nuestros museos y exponer su accesibilidad para uso público (Bertacchini y Morando, 2013: 60-72; Robles-Ortega et al., 2012: 326-331; Sylaiou et al., 2010: 243-253). La primera fase de este proyecto se realizó en el Museo Arqueológico Nacional (Madrid). Consistió en el fotografiado de medio centenar de epígrafes romanas de diversa tipología, de las cuales pudieron ser modeladas en 3D con éxito solo37 de ellas. La segunda etapa se realizó en 2015, en el Museo Nacional de Arte Romano (Mérida), con más avances en la aplicación práctica de esta técnica para la digitalización del patrimonio epigráfico. Debemos mencionar además que realizamos la modelización en 3D con dos softwares diferentes comparativamente para establecer ventajas y desventajas de

\footnotetext{
${ }^{4}$ Las numerosas aplicaciones de esta técnica innovadora ha sido presentada en una sesión monográfica desarrollada en el Museo Arqueológico Nacional (Madrid), el 16 de junio de 2016, organizada por el MAN y el Centro CIL II-Universidad de Alcalá, http://www.man.es/man/actividades.
} 
cada $u o^{5}$. En este lapso se han fotografiado 75 inscripciones romanas, de variada tipología, y de las cuales se adquirieron 59 modelos en 3D. Sumando ambas fases, el proyecto Epigraphia 3D ha logrado digitalizar y modelar en 3D un total de 96 epígrafes romanas, cifra más alta alcanzada, no solo en España sino a nivel mundial, hasta la actualidad. (RamírezSánchez et al., 2015: 371-396) ${ }^{6}$.

El empleo de tecnologías de bajo coste como Structure from Motion (SfM) e Image Based Modeling (IBM) no supone que la utilización del láser escáner para la digitalización de inscripciones esté superada, ni mucho menos, pues para algunos proyectos se requiere una malla más compleja, por lo cual esta tecnología sigue siendo indispensable. Además, como explicaremos en el siguiente apartado, en la mayoría de las inscripciones en 3D digitalizadas con la técnica SfM, el trazado de las letras no se traslada a la malla del objeto, por lo que hay que recurrir a vías de exploración de técnicas mixtas, que combinen la utilización del láser escáner y/o la digitalización de un vaciado, para poder obtener modelos perfectamente resueltos.

Entre los proyectos más recientes que han utilizado láser escáner en epigrafía se encuentra el Visionary Cross Project, impulsado por un equipo de investigadores del Istituto di Scienza e Tecnologie dell'Informazione "A. Faedo" (ISTI) del Consiglio Nazionale dell Ricerche (CNR), que ha digitalizado la célebre Cruz de Ruthwell, que se conserva en la iglesia escocesa del mismo nombre, modelándola en 3D y posibilitando la lectura y transcripción del texto en escritura rúnica escrito sobre el soporte (Leoni et al., 2015: 5.1-5.22). En dicho proyecto se ha empleado un láser escáner de triangulación Minolta Vivid 910. El trabajo de campo se extendió durante tres días para obtener 350 capturas necesarias para el procesamiento posterior de los datos en trabajo de laboratorio ${ }^{7}$.

\section{DIGITALIZACIÓN Y MODELADO 3D DE LAS INSCRIPCIONES ROMANAS DE} AUGUSTA EMERITA

La Epigrafía, junto con la Arqueología, es uno de los campos de investigación más activos en el campo de la investigación histórica, sobre todo de la Edad Antigua, ya que proporciona nuevos testimonios escritos que ayudan a los historiadores a avanzar en el estudio de la historia, la sociedad, las instituciones o la economía, por citar solo unos ejemplos, de culturas ya desaparecidas como la griega o la romana. España posee un rico y abundante

\footnotetext{
${ }^{5}$ En esta misma línea, recientemente hemos realizado un estudio similar sobre un lote de inscripciones romanas procedentes de la provincia de Bética, que se conservan en el Museo Arqueológico Nacional (Ramírez Sánchez et al., en prensa).

${ }^{6}$ Esta colección de inscripciones en 3D puede verse en las Galerías del proyecto Epigraphia 3D, accesible desde http://www.epigraphia3d.es/.

${ }^{7}$ Más información en la página web de difusión del proyecto: http://www.visionarycross.org/.
} 
patrimonio epigráfico de época romana en sus museos arqueológicos, tanto nacionales como provinciales. Este hecho, unido a una larga tradición en la investigación epigráfica, así como el estudio de esta materia en las Facultades de Letras de nuestro país desde comienzos del siglo XX y, desde la segunda mitad del siglo XIX, en la desaparecida Escuela Superior de Diplomática, tradicionalmente ha colocado a nuestro país en una privilegiada posición para el progreso de la investigación epigráfica.

En 2014, aprovechando la inminente reapertura del Museo Arqueológico Nacional (Madrid), después de una profunda renovación de su infraestructura y de su discurso museográfico, afrontamos el reto de abrir una nueva vía en el campo de la experimentación en la digitalización de inscripciones, apostando por una tecnología de bajo coste, tanto en los requisitos de infraestructura como de tipo invertido en su ejecución. En marzo de aquel año, cuatro semanas antes de la reapertura oficial del Museo, realizamos el trabajo de digitalización de 43 inscripciones romanas, entre piezas expuestas en sus colecciones como otras procedentes de sus almacenes. En su mayoría eran inscripciones hechas en mármol, aunque también se fotografiaron soportes realizados en piedra caliza, granito o arcilla, así como inscripciones realizadas sobre bronce (por ejemplo, una tessera hospitalis, una tabula y varios sellos).

Era la primera vez que se acometía un trabajo de esa naturaleza, no solo en nuestro país, sino a escala mundial. Aunque partíamos de una fase de experimentación previa desarrollada en Canarias sobre inscripciones del siglo XVIII, debemos reconocer que no preveíamos que los resultados fuesen a ser tan espectaculares.

Como hemos explicado (Ramírez-Sánchez et al., 2014: 467-474), optamos por la técnica denominada IBM que está relacionada con SfM, que consta de cinco fases, detalladas a continuación.

\subsection{Toma de fotografías de la inscripción}

El trabajo debe realizarse preferiblemente con luz natural (blanca) y en un lugar que permita la movilidad alrededor de la pieza, que debe permanecer inmóvil durante todo el proceso. Por término medio, se requieren entre 20 y 30 fotografías por inscripción, aunque algunas piezas con una geometría más compleja requieren un mayor número de fotografías y otras, más sencillas, requieren menos de 20. Para la realización de las fotografías hemos utilizado una cámara réflex digital Canon EOS 5DMark II en modo manual. En 2014, en el Museo Arqueológico Nacional, las piezas que se reprodujeron en la sala 20 de la primera planta, conocida como patio romano, son las que resultaron mejores, mientras que las 
inscripciones que se fotografiaron en sus salas sin posibilidad de trasladarlas, tuvieron menor calidad, con tonos anaranjados absorbidos del tipo de focos de iluminación utilizados ${ }^{8}$.

En el Museo Nacional de Arte Romano (MNAR, Mérida) el trabajo de exposición se realizó en la sala III de la planta superior, que posee características de iluminación natural excepcionales para la realización de este tipo de trabajo. Por lo cual, se procedió a cerrar la sala para evitar el acceso del público el personal del Museo habilitó una mesa para instalar las inscripciones a ser retratadas, trasladadas por los conservadores desde sus lugares de exhibición. Solo una pequeña parte de las inscripciones del MNAR modeladas en 3D debieron ser fotografiadas sin ser movidas, debido a su tamaño ${ }^{9}$. Por su parte, las dos cupae funerarias situadas en los jardines de la planta inferior, junto a la calzada romana, se fotografiaron también in $\operatorname{situ}^{10}$.Una mención especial merece el caso del monumento funerario de Zosimus que, por su complejidad, debió fotografiarse también en su emplazamiento original, en la planta baja del Museo, utilizando una escalera de gran altura para retratar su parte superior ${ }^{11}$.

\subsection{Procesamiento fotográfico con herramienta de modelado 3D}

En la misma fase de trabajo de campo, es recomendable que se realice una modelización de prueba de las fotografías realizadas, con el fin de comprobar si la labor ha sido bien realizada o si es necesario repetir las tomas del objeto. Para el modelado 3D hemos utilizado un ordenador portátil Asus A53S, que también hemos utilizado para las fases del trabajo de renderización y edición de la malla. El equipo informático utilizado poseía las siguientes características técnicas: procesador Intel Core i5-2410M (2x2,30 Ghz / $3 \mathrm{Mb}$ caché), 4 Gb de memoria RAM, HDD de 500 GB (5400 rpm), pantalla de 15,6" con resolución de1366 x 768, y tarjeta gráfica nVidia GT 520M de 512 MB GDDR3 (dedicada).

Hemos utilizado Autodesk 123D Catch, una excelente alternativa de software libre para el modelado 3D, cuyos óptimos resultados pudimos comprobar en la tarea realizada en el

\footnotetext{
${ }^{8}$ Es el caso, por ejemplo, de las inscripciones $n^{\circ} 12$ y $n^{\circ} 13$, fotografiadas en la sala 21 de la primera planta, o el miliario de Jaén ( $n^{\circ} 33$ ), fotografiado en la sala 18 de la misma planta. Las inscripciones pueden verse en la galería de inscripciones en 3D del Museo Arqueológico Nacional en la página web del proyecto: http://www.epigraphia3d.es/galeriacutea-3d-man.html.

${ }^{9}$ Es el caso, por ejemplo, de los relieves-retrato funerario de Lutatia Lupata ( $\left.\mathrm{n}^{\circ} 38\right)$, de L. Antestius Persicus ( $\mathrm{n}^{\circ}$ 39 ) o de Vettius Poponianus y su esposa $\left(n^{\circ} 40\right)$, que debieron fotografiarse en su emplazamiento de exhibición en el Museo, fijados a la pared. Las aras de gran tamaño o el pedestal de estatua también se fotografiaron en sus salas de exposición, en la planta baja, aunque debieron ser movidos con grúas para situarlos en el lugar que tuviera mejor luz natural través de los patios de iluminación del Museo.

${ }^{10}$ Son las inscripciones $n^{\circ} 42$ (http://www.epigraphia3d.es/3d-42.html) y $n^{\circ} 43$ (http:// www.epigraphia3d.es/3d43.html).

${ }^{11}$ Se trata de un monumento funerario realizado con bloques de granito de gran tamaño, todos ellos de forma rectangular, dispuestos en tres cuerpos escalonados, cuyo tamaño disminuye en altura. El último cuerpo está realizado con un solo bloque, dispuesto en posición vertical, en cuya parte superior se sitúa la inscripción funeraria, una placa de $26 \mathrm{~cm}$ de lado, encastrada en el bloque de granito. La altura total del monumento alcanza 2,65 metros y la base del cuerpo inferior, el más grande de todos, tiene 1,60 metros de lado. La inscripción que se exhibe en el monumento es una réplica realizada en yeso. La inscripción original se conserva en los almacenes del Museo y también fue fotografiada y se reproduce en $3 \mathrm{D}$ en el proyecto $\left(\mathrm{n}^{\circ} 47\right)$.
} 
Museo Arqueológico Nacional (Ramírez Sánchez et al., 2014: 467-474). También hemos empleado el programa Agisoft Photoscan, alternativa de software comercial ${ }^{12}$, el uso de uno u otro software varía según las necesidades del epigrafista digital, así como de disponer o no de acceso a la red; debido a que al utilizar Autodesk 123D Catch, es necesario el acceso a internet, pues el trabajo se realiza en la nube.

Debemos agregar que los resultados finales a obtener variarán según se utilice uno u otro software, así como también, el tiempo invertido en la generación de mallas en 3D. Pese a que se han realizado algunos estudios comparativos entre diferentes opciones de software aplicados a inscripciones romanas (Mittica et al., 2014: 401-420), la muestra es tan reducida que recientemente hemos efectuado una experimentación más amplia que favorezca a confirmar el potencial de Autodesk 123D Catch y Agisoft Photoscan para la investigación y difusión del patrimonio epigráfico (Ramírez-Sánchez et al., en prensa).

\subsection{Edición y reparación de la malla del objeto}

Con la obtención del modelo 3D a partir del procesamiento de las fotografías digitales, utilizando Autodesk 123D Catch, Agisoft Photoscan u otro programa alternativo, se obtiene una malla o superficie basada en triángulos que representa la geometría tridimensional del soporte epigráfico. Según hayamos empleado un software u otro, la malla tendrá un mayor o menor número de puntos, lo que influye no solo en la calidad del producto final, sino también en el tiempo que se debe invertir en esta tercera fase del trabajo. En esta etapa deberemos comprobar minuciosamente la malla del objeto, con el fin de corregir aquellas imperfecciones producidas en la fase anterior del trabajo. También hay que procurar especial atención a la adaptatividad de la malla, esto es el requisito de poseer una superficie adecuada en relación al número de puntos y triángulos que la componen, así como a la propia singularidad del objeto (Suárez et al., 2001: 903-910).

Para realizar el trabajo de edición y reparado de la malla hemos dispuesto de Blender, programa libre de código abierto, que goza de muchas aplicaciones en el campo de la digitalización del patrimonio histórico. Esta etapa es muy laboriosa, supone una mayor inversión de tiempo y en el caso de algunas inscripciones requiere muchos minutos, como explicaremos con detalle más abajo. Considerando que las inscripciones se fotografían sobre una superficie que posteriormente debe ser eliminada, asimismo, hay que cerrar la malla del soporte epigráfico, por el punto de apoyo de la inscripción sobre el suelo que no ha sido

\footnotetext{
${ }^{12}$ Autodesk 123D Catch es producido por CAD/CAM Software, con sede central en San Rafael (California, USA), http://www.123dapp.com/. Agisoft Photoscan es producido por Agisoft LLC, con sede central en San Petersburgo (Rusia), http://www.agisoft.com/. Este programa tiene una licencia para uso educativo, que tiene menor coste que la licencia comercial.
} 
fotografiado en la primera fase del trabajo. Por ello, nosotros disponemos de un plano simulado que seccionamos con la misma forma que solicita la malla del objeto, con el propósito de cerrar la geometría topológica del objeto.

\subsection{Tratamiento de texturas con un editor de gráficos rasterizados}

Cuando se realiza la exportación del modelo tridimensional en la segunda fase del trabajo, se obtiene un archivo en formato JPG que contiene las texturas del soporte epigráfico. El tratamiento de esta trama debe realizarse con un editor de gráficos rasterizados. Por esto, hemos utilizado Photoshop CS4; sin embargo, en esta etapa puede emplearse cualquier software alternativo de código abierto como, por ejemplo, GIMP ${ }^{13}$.

El tratamiento de la textura permite modificar algunos parámetros para optimizar el grado de realismo del modelo 3D definitivo, así como enmendar problemas derivados de malas condiciones de iluminación al fotografiar el epígrafe, dentro de los límites ofrecidos por el software. Es necesario recalcar que, en este paso, será imposible reformar el resultado final de una textura deficiente provocada por la deficiente iluminación al momento de fotografiar en la primera fase del trabajo.

Habría que decir también que del archivo en formato JPG extraemos la textura necesaria para el plano simulado generado en la fase anterior; no obstante, es importante otorgarle un color diferenciado al resto de la inscripción, con el fin de hacer saber, a quien observe el modelo 3D, que el lado observado es una restitución no un sector original de la inscripción. De igual manera, el tono empleado no debe alejarse demasiado de la gama cromática de la piedra original, para no generar excesivo ruido.

\subsection{Publicación y difusión en Internet}

El trabajo concluye con la publicación en internet de las inscripciones romanas en 3D, para permitir su uso a los públicos objetivos del proyecto. Para hacerlo, contamos con varias opciones. La más rápida y, a la vez, la más económica, es publicar los objetos en plataformas como Sketchfab ${ }^{14}$, Verold Studio o $\mathrm{p} \mathrm{d}^{15}$, que no solo permiten publicar y compartir los objetos tridimensionales, sino también crear estrategias de difusión en la web social. De todas ellas, la que más ha crecido en los últimos años ha sido Sketchfab, gracias a su facilidad de manejo y a las herramientas que ofrece a los usuarios. Estas plataformas utilizan la tecnología WebGL, un estándar desarrollado por un consorcio tecnológico llamado Khronos Group, que

\footnotetext{
${ }^{13}$ GIMP-GNU Image Manipulation Program, http://www.gimp.org.

${ }^{14}$ Véase https://sketchfab.com/.

${ }^{15}$ Véase https://p3d.in/.
} 
permite la visualización web de objetos tridimensionales GPU sin necesidad de instalar ningún plugin en el ordenador.

Frente a las alternativas de estas plataformas propietarias, podemos contar con desarrollos específicamente creados para el patrimonio digital, como el framework 3DHOP (3D Heritage Online Presenter), generado por un equipo de investigadores del Visual Computing Lab del ISTI-CNR de Pisa (Potenziani et al., 2015: 129-141) ${ }^{16}$. Se trata de un marco para el progreso de la visualización web del patrimonio histórico, desde edificios y yacimientos arqueológicos, hasta objetos de pequeño tamaño, que sean escalables y modulares. Sus desarrolladores afirman que cualquier usuario con conocimientos de programación en JavaScript y en diseño web no debería tener problemas para manejar este framework, aunque sí se requiere cierto nivel de entendimiento para implementar procesos más avanzados.

Respecto a los formatos de publicación de los objetos 3D, el más extendido es el OBJ, desarrollado por Wavefront Technologies, que se ha convertido en un estándar; aunque existen otras opciones como PLY (Polygon File Format) ${ }^{17}$ que son aceptados para publicar materiales arqueológicos en algunas publicaciones científicas (Ramírez-Sánchez et al., 2014: 472-473). En los próximos años asistiremos a una auténtica explosión en la presencia de imágenes en $3 \mathrm{D}$ en formatos de publicaciones electrónicas, mediante herramientas de visualización integradas en las propias plataformas que alojan las revistas electrónicas, como insertados en el propio PDF mediante el formato U3D. Hasta el momento, estas posibilidades son ofertadas por pocas revistas científicas en el campo de las Humanidades, como una apuesta de futuro, de algunos grupos editoriales, como Elsevier.

\section{DISCUSIÓN DE LOS RESULTADOS OBTENIDOS}

El trabajo de digitalización y modelización 3D de las inscripciones romanas del Museo Nacional de Arte Romano ha supuesto un progreso importante respecto al realizado en el Museo Arqueológico Nacional en 2014, tanto al duplicar el número de modelos obtenidos como al disminuir el tiempo de realización de las labores de edición y corrección de la malla con Blender, evidenciando la experiencia acumulada. Más aún, se han digitalizado con éxito soportes epigráficos, que no habíamos documentado anteriormente, al igual que inscripciones en bronce. Como se ha explicado en el apartado anterior, en la fase de modelado 3D hemos utilizado Autodesk 123D Catch y Agisoft Photoscan.

\footnotetext{
${ }^{16}$ Véase http://3dhop.net/.

${ }^{17}$ Revistas tan prestigiosas como Journal of Archaeological Science llevan ya varios años publicando artículos con imágenes en 3D. Sobre las especificaciones técnicas que requieren estos formatos para ser publicados en esta revista de la editorial Elsevier: https://www.elsevier.com/books-and-journals/content-innovation/3d-models.
} 


\begin{tabular}{|c|c|c|c|c|c|}
\hline Referencia & Fotografías & A. Photoscan $®$ & A. 123D Catch $®$ & Blender ${ }^{\circledR}$ & Tiempo total \\
\hline $3 \mathrm{D}-38$ & 25 & 40 & 0 & 255 & 320 \\
\hline 3D-39 & 25 & 60 & 0 & 210 & 295 \\
\hline $3 \mathrm{D}-40$ & 25 & 60 & 0 & 320 & 405 \\
\hline $3 D-41$ & 25 & 45 & 0 & 355 & 425 \\
\hline $3 D-42$ & 20 & 40 & 0 & 245 & 305 \\
\hline $3 D-43$ & 20 & 40 & 0 & 95 & 175 \\
\hline $3 D-44$ & 15 & 35 & 0 & 65 & 115 \\
\hline $3 D-45$ & 15 & 40 & 0 & 85 & 140 \\
\hline $3 D-46$ & 60 & 600 & 0 & 345 & 1005 \\
\hline $3 D-47$ & 15 & 35 & 0 & 90 & 140 \\
\hline $3 D-48$ & 25 & 0 & 20 & 135 & 180 \\
\hline $3 \mathrm{D}-49$ & 25 & 40 & 0 & 75 & 140 \\
\hline $3 \mathrm{D}-50$ & 20 & 40 & 0 & 130 & 190 \\
\hline $3 D-51$ & 20 & 40 & 0 & 90 & 150 \\
\hline 3D-52 & 25 & 40 & 0 & 85 & 150 \\
\hline $3 D-53$ & 15 & 0 & 15 & 65 & 95 \\
\hline $3 D-54$ & 15 & 30 & 0 & 75 & 120 \\
\hline $3 D-55$ & 15 & 40 & 0 & 75 & 130 \\
\hline $3 D-56$ & 15 & 35 & 0 & 90 & 140 \\
\hline $3 D-57$ & 15 & 30 & 0 & 105 & 150 \\
\hline 3D-58 & 15 & 30 & 0 & 75 & 120 \\
\hline 3D-59 & 20 & 40 & 0 & 75 & 135 \\
\hline $3 D-60$ & 20 & 0 & 20 & 80 & 120 \\
\hline 3D-61 & 15 & 30 & 0 & 85 & 130 \\
\hline $3 D-62$ & 20 & 40 & 0 & 160 & 220 \\
\hline 3D-63 & 15 & 30 & 0 & 70 & 115 \\
\hline 3D-64 & 25 & 40 & 0 & 75 & 140 \\
\hline $3 D-65$ & 25 & 40 & 0 & 95 & 160 \\
\hline $3 D-66$ & 25 & 40 & 0 & 190 & 255 \\
\hline 3D-67 & 25 & 40 & 0 & 100 & 165 \\
\hline $3 D-68$ & 20 & 40 & 0 & 90 & 150 \\
\hline 3D-69 & 20 & 40 & 0 & 70 & 130 \\
\hline 3D-70 & 20 & 40 & 0 & 100 & 160 \\
\hline 3D-71 & 15 & 30 & 0 & 65 & 110 \\
\hline 3D-72 & 15 & 35 & 0 & 95 & 145 \\
\hline 3D-73 & 15 & 30 & 0 & 60 & 105 \\
\hline 3D-74 & 15 & 30 & 0 & 75 & 120 \\
\hline 3D-75 & 15 & 30 & 0 & 60 & 105 \\
\hline 3D-76 & 25 & 40 & 0 & 85 & 150 \\
\hline 3D-77 & 15 & 30 & 0 & 70 & 115 \\
\hline 3D-78 & 15 & 35 & 0 & 90 & 140 \\
\hline 3D-79 & 15 & 30 & 0 & 70 & 115 \\
\hline 3D-80 & 20 & 35 & 0 & 100 & 155 \\
\hline 3D-81 & 15 & 35 & 0 & 120 & 170 \\
\hline 3D-82 & 15 & 35 & 0 & 110 & 160 \\
\hline 3D-83 & 15 & 40 & 0 & 85 & 140 \\
\hline 3D-84 & 15 & 35 & 0 & 70 & 120 \\
\hline 3D-85 & 15 & 30 & 0 & 70 & 115 \\
\hline 3D-86 & 20 & 40 & 0 & 90 & 150 \\
\hline 3D-87 & 20 & 35 & 0 & 90 & 145 \\
\hline 3D-88 & 20 & 40 & 0 & 110 & 170 \\
\hline 3D-89 & 15 & 30 & 0 & 70 & 115 \\
\hline 3D-90 & 15 & 35 & 0 & 75 & 125 \\
\hline 3D-91 & 15 & 0 & 15 & 75 & 105 \\
\hline 3D-92 & 20 & 5 & 0 & 100 & 155 \\
\hline 3D-93 & 20 & 0 & 20 & 95 & 135 \\
\hline 3D-94 & 20 & 35 & 0 & 90 & 145 \\
\hline 3D-95 & 15 & 40 & 0 & 95 & 150 \\
\hline 3D-96 & 20 & 40 & 0 & 90 & 150 \\
\hline
\end{tabular}

Tabla 1. Digitalización y modelización 3D de las inscripciones romanas del MNAR: número de fotografías, tiempo (en minutos) del modelado en Agisoft Photoscan o en Autodesk 123D Catch, renderización con Blender y tiempo total de trabajo invertido en cada inscripción.

En la Tabla 1 recogemos los datos del proceso de fotografiado, modelado y renderización de cada una de las 59 inscripciones romanas del MNAR que se pueden ver en 
la web del proyecto, así como en la aplicación para dispositivos móviles. Como ya hemos explicado en otro trabajo (Ramírez Sánchez et al., en prensa), este tipo de trabajos experimentales pueden ser de interés para los futuros epigrafistas que vayan a realizar trabajos de este tipo, pero también para los museos arqueológicos, ya que les pueden permitir programar, con bases fiables, cuál es el tiempo que se puede invertir en los trabajos de digitalización y modelización 3D de sus fondos epigráficos. Todo ello sin perder de perspectiva que las futuras versiones del software que hemos utilizado, así como otros nuevos productos, pueden rebajar sensiblemente los tiempos de procesado y de edición de las inscripciones.

Como se puede apreciar en la Tabla 1, el tiempo medio por cada inscripción se sitúa en casi tres horas de trabajo, incluyendo el tiempo invertido en la realización de las fotografías en el propio museo, aunque algunas inscripciones han necesitado una mayor inversión de tiempo, tanto debido a la complejidad de su geometría como al tiempo necesitado para su fotografiado. Se constata que, en algunas inscripciones, como el fragmento superior de la estela de cabecera recta que contiene el epitafio de Ebrilia (3D-89) y las aras funerarias de Iulia Caesiana (3D-91), entre otras, han supuesto una inversión de tiempo de trabajo por debajo de la media. En algunos casos, influye menos el factor tamaño del soporte, que la complejidad geométrica o topológica del propio soporte epigráfico. En otros términos, la placa funeraria que contiene el epitafio de Cn. Emilius lucundus (3D-63), a pesar de su gran tamaño, ha supuesto una inversión de tiempo de trabajo igual a otras inscripciones de tamaño mucho menor, aun cuando presentaban singularidades en su malla, por la existencia de decoración en relieve, como sucede con la célebre placa funeraria de Sentia Amarantis (3D-44).

En cambio, las inscripciones que han requerido un mayor esfuerzo, sobre todo en la edición y corrección de la malla con el software Blender, han sido los relieves-retrato funerarios, como el de Vettius Pomponianus y su esposa Casia Felicissima (3D-40), la tabula hospitalis de bronce (3D-41) y el monumento funerario de Zosimus, beneficiarius de la legión VII Gemina (3D-46). Este último necesitó varias horas de trabajo para fotografiarlo, además de las necesarias para seleccionar las 60 fotografías que posteriormente fueron necesarias procesar, más las casi 6 horas de trabajo de edición y corrección de la malla con Blender.

\section{DIFUSIÓN DE LAS INSCRIPCIONES ROMANAS DE AUGUSTA EMERITA EN 3D: DE LA WEB A LOS DISPOSITIVOS MÓVILES}

Como ya hemos explicado anteriormente, la difusión de los modelos 3D a través de internet, requiere la utilización de herramientas que cumplan el estándar WebGL, un producto lanzado en 2011 por el Grupo Khronos, que en muy poco tiempo se ha convertido en el estándar para la visualización multiplataforma de gráficos 3D. Existen alternativas rápidas y económicas como Sketchfab, una plataforma social que permite publicar y compartir cualquier 
formato de archivo y que está siendo utilizada, de forma experimental, por numerosos museos arqueológicos, algunos de ellos tan importantes como el propio British Museum ${ }^{18}$.

Un proyecto español que cabe destacar aquí es el Museo Virtual de Los Bañales, presentado en la UNED-Tudela en abril de 2016, en la actualidad alcanza la cifra de 42 objetos digitales, entre inscripciones, estructuras arquitectónicas, esculturas y otros restos materiales procedentes del yacimiento romano de Los Bañales (Uncastillo, Zaragoza) y alrededores ${ }^{19}$. Todos estos ejemplos que hemos glosado aquí permiten la visualización de estos recursos, así como su utilización compartiéndolos en redes sociales, blogs, etc., pero no permiten su descarga para poder imprimir los modelos en una impresora 3D. Esta es una vía que está aún por explorar por los museos e instituciones españolas. En el Reino Unido, por ejemplo, el consorcio UN Digital Heritage permite la descarga, previo pago, de su colección de modelos 3D, tanto para uso personal como comercial ${ }^{20}$.

El proyecto Epigraphia 3D ofrece, desde el año 2014, acceso a una selección de inscripciones romanas de las diferentes provincias de la Hispania romana, dentro de un proyecto de difusión de la cultura clásica entre estudiantes de Educación Secundaria y Bachillerato y también entre el público en general. La página web del proyecto permite acceder a las galerías 3D en las que están recogidas casi cien inscripciones romanas procedentes de los dos grandes museos de referencia en su campo en España ${ }^{21}$. Además, incluye materiales didácticos creados para introducir a los usuarios de la web en la sociedad, la cultura y las instituciones romanas, permitiendo así profundizar en las preguntas que tantas veces hemos planteado los estudiosos de estos testimonios escritos: ¿quién? ¿para quién? ¿por qué? ¿cómo? ¿cuándo? ¿dónde?

Las Tecnologías de la Información y la Comunicación ayudan a relacionar el ingente caudal de información que tenemos sobre estas inscripciones, pero sobre todo ayudan a hacer más fácil la visualización de la información. Uno de los aspectos en los que esto es más evidente es en el campo de la dispersión geográfica de los hallazgos. A través de herramientas gratuitas, de uso tan extendido entre los jóvenes y el público en general como Google Maps, hemos relocalizado sobre el mapa, la procedencia de aquellas inscripciones de ambos Museos cuya procedencia segura conocemos.

\footnotetext{
${ }^{18}$ Véase https://sketchfab.com/britishmuseum. En España, uno de los museos que ha sabido aprovechar mejor el potencial de estos recursos y su difusión en la plataforma Sketchfab es el Museo d'Arqueologia de Catalunya, que actualmente, dispone de 96 objetos digitales en 3D en su galería: https://sketchfab.com/macb3d. Otro ejemplo en España, aunque algo más alejado de las cifras de objetos del MAC es la colección en 3D del Museo de Pontevedra, que cuenta con 34 modelos en 3D: https://sketchfab.com/museodepontevedra. En Portugal, el Museo Cidade de Ammaia cuenta ya con una colección de 15 restos arqueológicos en 3D, https://sketchfab.com/ammaia.

${ }^{19}$ El proyecto Museo Virtual de Los Bañales está dirigido por Javier Andreu Pintado (Universidad de Navarra), los trabajos de digitalización y modelización 3D han sido realizados por Pablo Serrano: http://www.losbanales.es/indexmuseo.html. Los objetos 3D se difunden a través de la plataforma Sketchfab: https://sketchfab.com/banalesmuseovirtual.

${ }^{20}$ Entre los objetos digitales se encuentra una selección de inscripciones romanas procedentes de diversos yacimientos cercanos al muro de Adriano: http://www.nu-digitalheritage.com/.

21 En http://www.epigraphia3d.es/.
} 
Por ejemplo, las cincuenta y nueve inscripciones de Augusta Emerita aparecen distribuidas sobre la cartografía actual de la ciudad de Mérida y su entorno. De un simple vistazo, es posible comprobar cuántas inscripciones funerarias han aparecido en las excavaciones arqueológicas desde el siglo XIX hasta nuestros días en su contexto original de exhibición, o muy cercano a él, ya que se aprecia sobre el mapa que han aparecido fuera del espacio de la antigua ciudad romana, mientras que otras inscripciones (posiblemente porque fueron reutilizadas en las construcciones de la ciudad en época medieval o moderna), han aparecido intramuros o en las propias murallas de la ciudad, como muestra esta imagen:

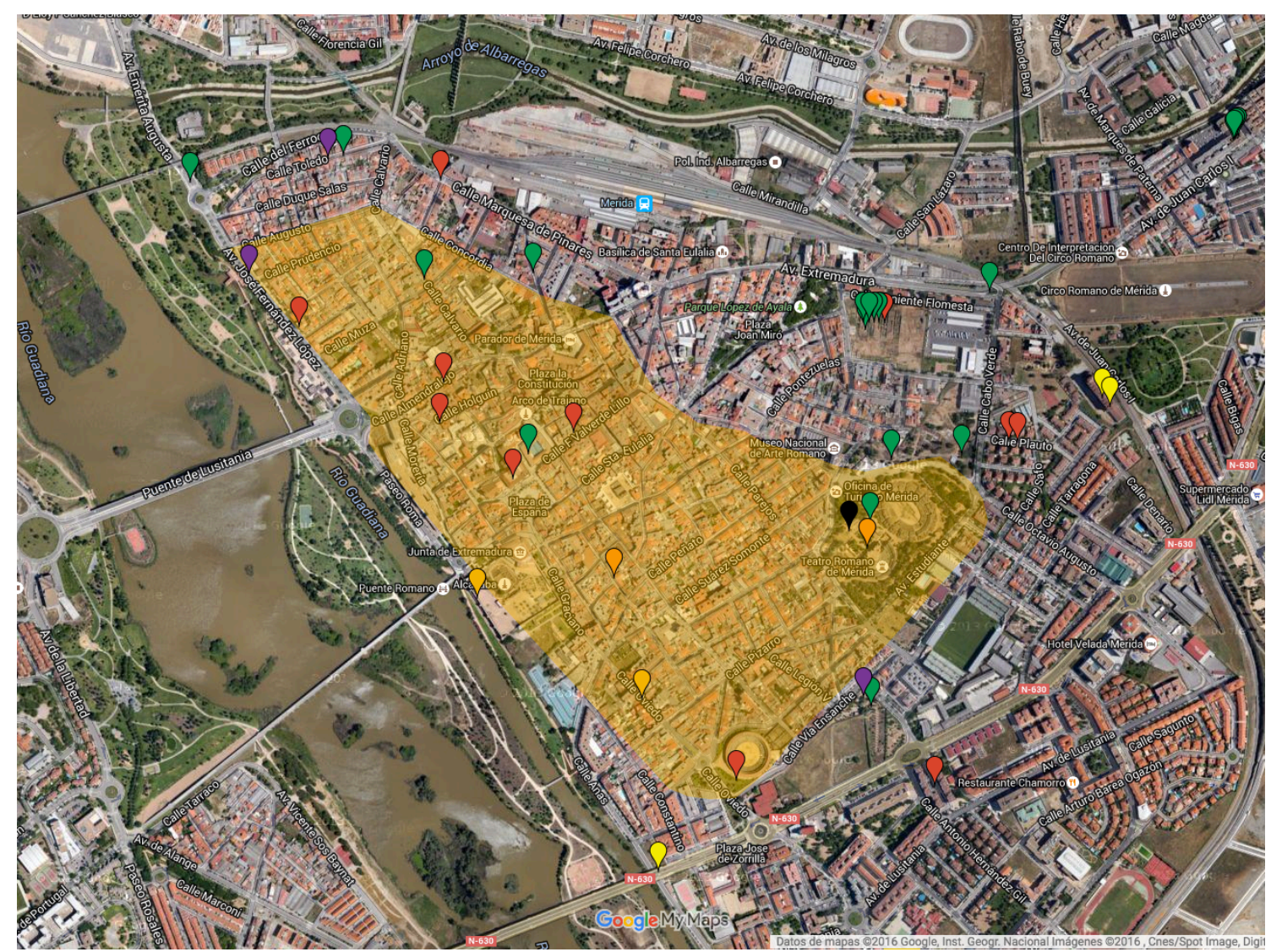

Figura 1. Mapa de dispersión geográficas de las inscripciones de Augusta Emerita, según su lugar de aparición sobre el callejero actual de Mérida y su entorno.

Sin embargo, el mayor reto que hemos afrontado con las inscripciones de Augusta Emerita en 3D ha sido acercarlas a los futuros visitantes del Museo Nacional de Arte Romano y a todos los amantes de la arqueología romana, a través de una aplicación desarrollada para dispositivos móviles que utilizan el sistema operativo Android. Es sabido que el acceso a internet a través de los dispositivos móviles (smartphones y tablets) se está incrementando de manera vertiginosa, en detrimento del habitual acceso desde los ordenadores de sobremesa o portátiles, y consideramos un reto tecnológico volcar toda la información de nuestro proyecto a una aplicación móvil de desarrollo propio. 
No vamos a detenernos aquí en los detalles técnicos de esta aplicación, sobre los cuales ya nos hemos ocupado en otro trabajo (Ramírez-Sánchez et al., en prensa), pero sí queremos destacar que se trata de la primera aplicación de su naturaleza que se ha realizado hasta la fecha a nivel mundial, lo que sitúa nuestro proyecto en la vanguardia internacional en este campo de la transferencia y la innovación científica en epigrafía digital ${ }^{22}$. En menos de $40 \mathrm{MB}$, es posible tener instalado en cualquier smartphone o tablet Android una aplicación que permite acceder, sin necesidad de conexión a la red, a las cincuenta y nueve inscripciones romanas en 3D del MNAR, así como a la geolocalización de los epígrafes sobre el actual callejero de Mérida y su entorno, un campo de indudable interés tanto para la protección y conservación del patrimonio histórico como para su explotación turística (Bollini et al., 2013: 481-493).

El equipo de desarrollo de esta aplicación móvil ha afrontado varios retos importantes, uno de ellos, ha sido la compresión de los modelos 3D, puesto que algunos de los objetos digitales más pesados alcanzaban los $8 \mathrm{MB}$; sin perder de vista, uno de los factores críticos de la aplicación, la calidad de las imágenes tridimensionales. El resultado final ha sido una aplicación en la que los objetos digitales 3D se cargan a gran velocidad y con una resolución de 4096 x 4096 píxeles, todo ello, sin superar el umbral de 2 MB en los modelos de mayor tamaño.

\section{CONCLUSIONES}

La tecnología 3D realizada con presupuestos ajustados y sin necesidad de un equipamiento avanzado es una realidad. En los próximos años el escenario seguirá modificándose a gran velocidad, a través de la introducción de programas que, de manera creciente, reduzcan la curva de aprendizaje a nuevos usuarios, sobre todo cuando las inscripciones a digitalizar no tengan especial complejidad, ni en su accesibilidad, así como tampoco en sus características geométricas. En este contexto, los proyectos de epigrafía digital ya existentes, como las bases de datos epigráficas que nacieron cuando nadie podía sospechar el desarrollo que iba a alcanzar la fotografía digital, tendrán que evolucionar a corto término, hacia entornos que adicionen imágenes de alta calidad; a mediano plazo, éstas deberán comenzar su progresiva reconversión incorporando la edición en TEI-XML y la explotación de los datos enlazados, empero, deberán incrementar formatos de representación en $3 \mathrm{D}$, como ya lo han iniciado a hacer los arqueólogos en sus parcelas de investigación (Bruno et al., 2010: 42-49; Galeazzi, 2016: 159-169; Remondino y Campana, 2014).

\footnotetext{
${ }^{22}$ La aplicación móvil Epigraphia 3D está disponible para su descarga en Google Play desde el 10 de mayo de 2016 y está optimizada para la versión 4.0.3 y posteriores del sistema operativo Android: https://play.google.com/store/apps/details?id=es.epigraphia3d.android.activities\&hl=es.
} 
Somos conscientes de que, en este contexto, uno de los aspectos cruciales será el costo monetario de los desarrollos tecnológicos, por ello, los desarrollos de bajo coste aparecen, aunque no renuncian a la calidad final del producto ni a su rigor científico. No cabe duda que entre los futuros retos que debemos alcanzar, se encuentra la búsqueda de alternativas de visualización $3 \mathrm{D}$, que cumpla los requisitos de la tecnología WebGL, y permitan visualizar los gráficos 3D en navegadores web multiplataforma, sin necesidad de instalar de ningún tipo de plugins.

Hasta el momento, plataformas como Sketchfab han sido una solución fácil y económica para proyectos como Epigraphia 3D o para nuestros museos e instituciones, sin embargo, debemos abordar nuevos escenarios, a través de frameworks como 3DHOP (Potenziani et al., 2015: 129-141).

Esperamos que actividades como las aquí evidenciadas, realizadas entre humanistas, ingenieros informáticos y geómatas, sirvan de estímulo para el impulso de equipos interdisciplinares que favorezcan a superar barreras entre nuestras disciplinas que, como todos sabemos, son más mentales que físicas. En este campo, como en otros de las Humanidades Digitales, apenas hemos comenzado a desbrozar un camino que se vislumbra largo, pero que debemos comenzar a transitar cuanto antes.

\section{REFERENCIAS BIBLIOGRÁFICAS}

BARMPOUTIS, A., BOZIA, E. y WAGMAN, R.S. (2010). "A Novel Framework for 3D Reconstruction and Analysis of Ancient Inscriptions". Journal of Machine Vision and Applications, 21.6, 989-998.

BERTACCHINI, E. y MORANDO, F. (2013). "The Future of Museums in the Digital Age: New Models for Access to and Use of Digital Collections". International Journal of Arts Management, 15.2, 60-72.

BODEL, J. (2012). "Latin Epigraphy and the IT Revolution". En Epigraphy and the Historical Sciences, J.K Davies y J.J. Wilkes (eds.), 275-296. Oxford: Oxford University Press.

BOLLINI, L., DE PALMA, R. y NOTA, R. (2013). "Walking into the Past: Design Mobile App for the Geo-Referred and the Multimodal User Experience in the Context of Cultural Heritage". Lecture Notes in Computer Science, 7973.3, 481-493.

BRUNO, F., BRUNO, S., DE SENSI, G., LUCHI, M.L. y MANCUSO, S. (2010). "From 3D Reconstruction to Virtual Reality: A Complete Methodology for Digital Archaeological Exhibition". Journal of Cultural Heritage, 11.1, 42-49. 
BUONOPANE, A., GROSSI, P., GUARNIERI, A. y PIROTTI, F. (2005). "L'impiego del laser scanner nel rilievo delle iscrizioni sui miliari”. En Colloquio internazionale AIEGL Bertinoro, I. Misurare il tempo, misurare lo spazio, M. G. Bertinelli Angeli y A Donati. (eds.), 373-388. Faenza, Ravenna: Fratelli Lega Editori.

CORREIA SANTOS, M.J., SOUSA, O. y PIRES, H. (2014a). "Nuevas lecturas de las inscripciones del santuario de Panóias (Vila Real, Portugal)". Sylloge Epigraphica Barcinonensis, 12, 197-224.

CORREIA SANTOS, M.J., SOUSA, O., PIRES, H., FONTE, J. y GONÇALVES-SECO, L. (2014b). "Travelling back in Time to Recapture Old Texts. The Use of Morphological Residual Model (M.R.M.) for Epigraphic Reading: Four Case Studies (CIL 02, 02395a, CIL 02, 02395c, CIL 02, 02476, CIL 02, 05067)". En Information Technologies for Epigraphy and Cultural Heritage. Proceedings of the First EAGLE International Conference. Collana Convegni 26, S. S. Orlandi, R. Santucci, V. Casarosa y P.M. Liuzzo (eds.), 437-454. Roma: Sapienza Università Editrice. Recuperado de https://www.eagle-network.eu/.../2015/01/Paris-Conference-Proceedings.pdf el $11 / 05 / 2017$

ELLIOTT, T. (2014). "Epigraphy and Digital Resources". En The Oxford Handbook of Roman Epigraphy, Ch. Bruun y J. Edmondson (eds.), 78-85. New York: Oxford University Press.

FUSTER MORELL, M. (2011). "Acción colectiva a través de las redes: Comunidades de Creación Online para la construcción de bienes públicos digitales". Redes.com: Revistas de estudios para el desarrollo social de la comunicación, 6, 229-248.

GALEAZZI, F. (2016). "Towards the Definition of Best Practices in Archaeology: Assessing 3D Documentation Techniques for Intra-Site Data Recording". Journal of Cultural Heritage, 17, 159-169.

LEONI, Ch., CALliERI, M., DELLEPIANE, M., O'DONNELL, D.P., DEL TURCO, R.R. y SCOPIGNO, R. (2015). "The Dream and the Cross: A 3D Scanning Project to Bring 3D Content in a Digital Edition". Journal on Computing and Cultural Heritage, 8.1, 5.1-5.22. LERMA, J.L. y MUIR, C. (2014). "Evaluating the 3D Documentation of an Early Christian Upright Stone with Carvings from Scotland with Multiples Images". Journal of Archaeological Science, 46, 311-318.

LIUZZO, P.M., ZANNI, A., MARTINELLI, L., LOSA, L. y DE NICOLAO, P. (2014). "The EAGLE Mediawiki. A Fully Collaborative Database for Academics, Data Engineers and the General Public". En Information Technologies for Epigraphy and Cultural Heritage. Proceedings of the First EAGLE International Conference. Collana Convegni 26, S. Orlandi, R. Santucci, V. Casarosa y P. M. Liuzzo (eds.), 187-201. Roma: Sapienza Università Editrice. Recuperado de http://archiv.ub.uni-heidelberg1 el 25/04/2017. 
MitTICA, D., PELlEGRINO, M. y ROCCO, A. (2014). "Low-Cost Structure from Motion Technology: An Open Approach for Epigraphical Digital Reconstruction". En Information Technologies for Epigraphy and Cultural Heritage. Proceedings of the First EAGLE International Conference. Collana Convegni 26, S. Orlandi, R. Santucci, V. Casarosa y P. M. Liuzzo (eds.), 401-420. Roma: Sapienza Università Editrice. Recuperado de http://archiv.ub.uni-heidelberg2 el 25/04/2017.

ORLANDI, S., SANTUCCI, R., CASAROSA, V. y LIUZZO, P.M. (eds.) (2014). Information Technologies for Epigraphy and Cultural Heritage. Proceedings of the First EAGLE International Conference. Collana Convegni 26. Roma: Sapienza Università Editrice. Recuperado de http://archiv.ub.uni-heidelberg.de/k/2337.pdf el 25/04/2017.

PIRES, H., FONTE, J., GONÇALVES-SECO, L., CORREIA SANTOS, M.J. y SOUSA, O. (2014). "Morphological Residual Model. A Tool for Enchancing Epigraphic Readings of Highly Erosioned Surfaces". En Information Technologies for Epigraphy and Cultural Heritage. Proceedings of the First EAGLE International Conference. Collana Convegni 26, S. Orlandi, R. Santucci, V. Casarosa y P. M. Liuzzo (eds.), 133-144. Roma: Sapienza Università Editrice. Recuperado de http://archiv.ub.uni-heidelberg3 el 25/04/2017

POTENZIANI, M., CALliERI, M., DELlePIANE, M., CORSINI, M., PONCHIO, F. y SCOPIGNO, R. (2015). "3DHOP: 3D Heritage Online Presenter". Computers \& Graphics, 52, 129-141.

RAMÍREZ-SÁNCHEZ, M. (2003). "Recursos de epigrafía y numismática en internet: balance actual y perspectivas en España". Boletín Millares Carlo, 22, 275-301.

(2010). "Epigrafía e Internet en España hoy: una introducción necesaria". En Cursos sobre Patrimonio Histórico, 14, J.M. Iglesias Gil (ed.), 15-41. Santander: Universidad de Cantabria.

(2016). "Las Ciencias y Técnicas Historiográficas en el contexto de las Humanidades Digitales: oportunidades para su desarrollo". En “Dicebamus hesterna die...". Estudios en homenaje a los profesores Pedro J. Arroyal Espigares y M. Teresa Martín Palma, Marchant Rivera A. y Barco Cebrián L. (eds.), 365-392. Málaga: Universidad de Málaga. Recuperado de http://riuma.uma.es/xmlui/Dicebamus hesterna die.pdf el 25/04/2017.

RAMÍREZ-SÁNCHEZ, M., GARCÍA SÁNCHEZ, M. y GIRALT SOLER, S. (2015). "Epigraphia 3D. Un proyecto de innovación científica en la divulgación del patrimonio epigráfico de Hispania”. Epigraphica, 77, 371-396.

RAMÍREZ-SÁNCHEZ, M., SUÁREZ-RIVERO, J.P. y CASTELLANO HERNÁNDEZ, M.Á. (2014). "Epigrafía digital: tecnología 3D de bajo coste para la digitalización de 
inscripciones y su acceso desde ordenadores y dispositivos móviles". El profesional de la información, 23.5, 467-474. Recuperado de https://recyt.fecyt.es/ el 25/04/2017.

RAMÍREZ-SÁNCHEZ, M., SUAREZ-RIVERO, J.P., TRUJILLO, A., FERNÁNDEZ, P., SANTANA, J.M. y ORTEGA, S. (en prensa). "Digital Epigraphy Heritage Made Simple: An Android App for Exploring 3D Roman Inscriptions".

REMONDINO, F. y CAMPANA, S. (eds.) (2014). 3D Recording and Modeling in Archaeology and Cultural Heritage. Theory and Best Practices. Oxford: Archaeopress (BAR International Series 2598).

ROBLES ORTEGA, M.D., FEITO, F.R., JIMÉNEZ, J.J. y SEGURA, R.J. (2012). "Web Technologies Applied to Virtual Heritage: An Example of an Iberian Art Museum". Journal of Cultural Heritage 13.3, 326-331.

SYLAIOU, S., MANIA, K., KAROULIS, A. y WHITE, M. (2010). "Exploring the Relationship Between Presence and Enjoyment in a Virtual Museum". International Journal of Human-Computer Studies, 68, 243-253.

SUÁREZ RIVERO, J.P., CAREY, G.F. y PLAZA, Á. (2001). "Graph Based Data Structures for Skeleton Based Refinement Algorithms". Communications in Numerical Methods in Engineering, 17.12, 903-910. 